\title{
Inteligencia Artificial y sociedad ¿El fenómeno social tecnológico 4.0?
}

\author{
doi: 10.52749/fh.v2i1.11
}

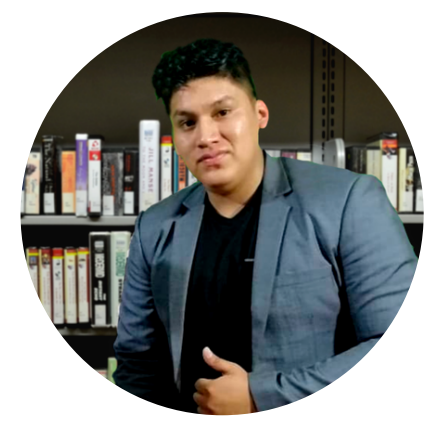

\section{KENNER MORI CASTRO}

CEO y cofundador de Innovacit, fundador de la organización social "Cultura Steam". Miembro del Consejo Regional de Ciencia, tecnología, emprendimiento e innovación de Huánuco (CORCITEI). Miembro del Instituto de Extrapolítica y Transhumanismo (IET). Formó parte del Consejo Regional de la Juventud Huánuco. Llevó estudios de Sociología en la Universidad Nacional Hermilio Valdizán, Maestría en Gestión Pública para el Desarrollo Social.

kenner.yesser@gmail.com

@kennermori19

Al usar el celular para hablar con nuestro asistente virtual o traducir palabras a diferentes idiomas mediante el habla hacemos uso del speech recognition; cuando generamos texto y noticias escritas nos referimos al natural language processing; al usar autos autónomos, el reconocimiento facial y la robótica inteligente entramos al campo del computer vision. Todo esto que parece ficción para muchas personas no es más que años de investigación científica e inversión en informática y sus distintas ramas. Lo grandioso de empresas como Google, IBM, Amazon, Microsoft, Facebook, Apple u Open Al, es que han puesto sobre la mesa discusiones que antes parecían utópicas y hoy sus avances son analizados desde distintas disciplinas académicas.

\section{El fenómeno social tecnológico 4.0}

En el desarrollo de nuestra historia se han originado sucesos transcendentales conocidos como Revoluciones Industriales (RIs) y en cada uno de ellas podemos identificar ciertas tecnologías o "Fenómenos Sociales Tecnológicos" (FST) que generaron cambios sustanciales en cada RI y jugaron un papel fundamental en la construcción de nuestra sociedad actual. En la primera RI el FST 1.0 fue la máquina de vapor, la cual desempeñó un rol clave, pues al calentar agua en una caldera se generaba presión y movía maquinarias grandes; en la segunda $\mathrm{Rl}$, las investigaciones y disputas de Tomas Alva Edison y Nikola Tesla, lo que se conoció como la "guerra de las corrientes", fueron las precursoras de la generación masiva de energía eléctrica (continua y alterna), a la cual consideraremos como el FST 2.0, esto trajo consigo la era de la cadena de montaje, producción en masa e invención de muchos artefactos como la bombilla incandescente, el teléfono, la radio, el vehículo eléctrico y más. En la tercera RI, la invención del transistor fue la base para el diseño y construcción del ordenador, además, la Guerra Fría conjuntamente con la carrera espacial desataron grandes avances científicos, entre ellos la creación, uso y posterior democratización del Internet. Las "Tecnologías de la Información" y la electrónica fueron los FST 3.0, fue el tránsito de lo analógico a lo digital.

En esta cuarta RI se han desarrollo nuevas tecnologías dentro de las cuales podemos destacar la inteligencia artificial (IA) cuya influencia creciente en el estilo de vida del ciudadano promedio me permite afirmar que estamos frente al "Fenómeno Social Tecnológico 4.0" (FST 4.0).

De manera preliminar definamos lo que es la IA y un Fenómeno Social. La IA es un modelo computacional que cuenta con la capacidad de imitar la inteligencia y el comportamiento humano. Por otro lado, los positivistas buscan los hechos o las causas de los fenómenos sociales con independencia de los estados subjetivos de los individuos, por esta razón, entenderemos por fenómeno social lo propuesto por Durkheim para quien los hechos o fenómenos sociales deben considerarse como "cosas" que ejercen una influencia externa sobre las personas (Arroyo Gonzales, 2006). Con los conceptos claros sobre estos dos términos llegamos a este nuevo FST 4.0, donde Machine Learning (Aprendizaje de máquinas) y los algoritmos denominados Deep Learning (Aprendizaje Profundo) la componen.

La IA tiene un impacto socioeconómico cada vez más significativo en diversos campos como la educación, la agricultura, las finanzas, el trabajo, la salud, el medio ambiente $y$, en consecuencia, en 
cada persona que se desenvuelve en estas áreas. Además, tiene el poder de generar soluciones rápidas y a gran escala como el desarrollo de robots inteligentes para múltiples sectores de la sociedad, algoritmos para ayudar a reducir el calentamiento global, identificar problemas complejos de salud, disminuir los índices de pobreza, contribuir al logro de grandes avances científicos, mejorar la transparencia de los gobiernos de cada país o en un aspecto diferente, dotar de poder a los gobiernos para el control de sus ciudadanos.

La evolución del Internet, el fenómeno del Big Data y el proceso de globalización, sentaron las bases para la digitalización de la información sobre la población y su historia. Con ello se entrenan algoritmos o modelos computacionales; ese es el caso de las "Redes Neuronales". Estos reciben, procesan y analizan la información, aprenden a realizar tareas por ensayo y error - como un niño cuando experimenta - son capaces de tomar mejores decisiones. Sin embargo, no toda la información en línea es confiable y nuestra cultura puede generar sesgos en los distintos modelos de machine learning que se desarrollen, esto manifiesta algunos de los retos a superar en este FST 4.0.

Cuando nos adentramos en este nuevo fenómeno comprendemos que las actitudes o pensamientos de muchas personas pueden ser influidos significativamente por ciertas empresas o grupos con intereses distintos y particulares. Para afectar lo que haces y piensas, algunos usan la información de los usuarios que les proveen las grandes empresas dedicadas a las redes sociales, a la búsqueda inteligente $\mathrm{o}$ al recojo de datos mediante la IA. De esta manera se puede manipular la actitud de los usuarios en distintos contextos, propiciar el debate, el odio hacia los demás y propagar pensamientos o teorías pseudocientíficas poco confiables. Esto debido a que los motores de búsqueda potentes nos proveen de recomendaciones, fake news, deepfakes e incluso influyen en las decisiones políticas, marchas, moda, partidos de futbol, éxitos de películas, violencia y el estallido de los problemas sociales existentes o nuevos. Es pertinente mencionar que aún es el inicio del FST 4.0 y en este artículo se abordarán ciertos alcances del mismo para un mejor análisis.

El inicio del desarrollo y programación de una IA se enmarca en intereses personales, políticos 0 empresariales. Esto supone que, quien tenga la IA más avanzada contará con mayor dominio y poder. Su fusión con otras tecnologías genera más incertidumbre, por ejemplo, no hay nada más peligroso que una IA cuente con información sobre tu personalidad, tus gustos, tus miedos, tus relaciones, tus amigos y tu localización a detalle. Con estos datos algunas empresas y gobiernos tienen el poder para predecir tu comportamiento, influir en la decisión que tomes el día de mañana respecto a tu trabajo, situación sentimental o cosas tan cotidianas como qué comprar o comer. El problema ya no es únicamente qué publicamos en las redes sociales o cómo usamos las apps, plataformas digitales o gadgets, sino que, por un "poder" con el que antes no contábamos y que ahora genera grandes soluciones, pero también supone peligros, parecemos títeres de alguien.

\section{El reto social frente al FST 4.0}

Las estructuras sociales, el trabajo y el empleo se van a ver a influenciadas por el FST 4.0, por tal motivo, uno de los retos de nuestra sociedad se encuentra en cómo hacer que su implementación y la automatización del empleo no deje atrás a las personas que tienen menos oportunidades de competir con una máquina que aprende o con personas más preparadas.

En nuestra sociedad actual la gran mayoría de personas que la sostienen son trabajadores con diferentes profesiones u oficios como profesores, enfermeros, contadores, mecánicos, periodistas, taxistas, barrenderos, entre otros. Muchos de ellos tienen un ingreso económico por debajo del promedio que los priva de lujos y de una buena educación. Sin caer en el tecno-pesimismo, si se automatizara gran parte de los trabajos $u$ oficios, el consumo de productos en el mercado bajaría; si no hay un adecuado ingreso económico, las personas limitarán y priorizarán las compras de algunos productos, sin mencionar el impacto en las personas con pobreza extrema. Esto impactaría en el bienestar social, psicológico y la calidad de vida de la población. Aunque son sucesos, quizás, un poco alejados o extremos debemos recordar que ya ocurrieron en cierta manera en las pretéritas RIs.

¿Cómo enfrentaremos la automatización y pérdida de empleo generado por este FST 4.0? El trabajo y la capacidad de trabajar proviene de nuestra educación, habilidades, destrezas e interacción social. Si se reemplaza gran parte de las actividades repetitivas, la ola de desempleo y falta de capacidades para ser empleado o crear empleo crecerá. En una era donde tener la capacidad de resolver problemas, crear soluciones e innovar es primordial, concluimos que la educación juega el rol más fundamental. Además, conjuntamente con 
políticas adecuadas podríamos encaminar el uso de la IA y evitar llegar a este "apocalipsis laboral". Figuras como Bill Gates y Mark Zuckerberg, dos de los responsables de la revolución tecnológica más grande de la historia, también consideran que el desempleo causado por la tecnología es un gran reto; incluso afirman que podría ser el conflicto mundial de este siglo (Oppenheimer, 2018).

Desde el ámbito jurídico podemos analizar 3 desafíos:

"Por un lado, cómo nos protegemos a nosotros mismos de los algoritmos inteligentes que nos reemplazan y superan en múltiples actividades. Por otro lado, cómo hacer para que esta nueva tecnología contribuya al desarrollo sostenible e incluso del ser humano. $Y$, por último, eventualmente, cómo se protegerán y se transformarán los derechos humanos en una transición que parece dirigirse hacia una simbiosis entre lo biológico, lo digital y lo artificial." (Corvalán, 2018, pág. 300)

Estos escenarios, donde el FST 4.0 tiene un protagonismo mayor en la sociedad, conllevan grandes retos políticos. Un ejemplo de esto es el futuro del transporte. Si mañana disminuyeran los costos de producción de los autos autónomos y fuese más accesible para las empresas ponerlas a disposición de la población, el servicio de taxis entraría en crisis. En cierta medida esto ya ocurrió con la aparición de la empresa Uber y otras similares. Por tal motivo, es importante que nuestras políticas de estado y de gobierno vayan de la mano con el avance científico y tecnológico, pero, sobre todo, posean un enfoque humanista en vez de ser dirigidos por algún tipo de ambición personal.

Cabe resaltar que en las tres anteriores revoluciones industriales se crearon más empleo e industrias que las que se destruyeron. Se generó mayor desarrollo socioeconómico y según el sistema político se logró mejores índices de desarrollo humano. No obstante, la cuarta RI es distinta y con la aparición de este FST 4.0 nos invita a reflexionar sobre si estamos educando a la nueva generación de manera correcta para el mercado de las siguientes décadas o para los trabajos futuros que podrían surgir. Según Oppenheimer (2018) las 10 áreas de trabajo del futuro serán: los asistentes de salud, los analistas de datos, ingenieros de datos y programadores, los policías digitales, los asesores de ventas, los cuidadores y programadores de robots, los profesores y maestros, los especialistas en energías alternativas, los artistas, deportistas y creadores de entretenimiento, los creadores y diseñadores de contenidos comerciales y por último los consejeros espirituales. Por ende, no hay duda de que el impacto e inevitable avance de la IA nos obligará a pensar y diseñar estrategias para todos los rubros sociales.

Por otro lado, el reto social venidero radicará en cómo enfrentaremos la llegada de la "Singularidad Tecnológica" (momento en el cual la IA iguala y supera la inteligencia humana) a fin de que una IA no reemplace en todo a un humano considerándolo inferior e innecesario; en ese contexto llegaríamos a un nivel superior del FST 4.0. Si bien es un suceso futurista, desde ahora debemos mejorar algunas habilidades que nos hacen humanos, tales como la creatividad, la ética y la innovación.

\section{GPT-3 ¿Una IA que escribirá por ti?}

Para ejemplificar cómo este FS 4.0 viene avanzando a pasos agigantados se tomará como ejemplo a GPT3, una de las IAs de Open Al que fue entrenada con millones de libros o artículos de internet. Esta Red Neuronal, que es parte del Procesamiento del Lenguaje Natural, genera textos con mucha coherencia, escribe líneas de código y diseña con solo recibir instrucciones. Entonces, suponemos que GPT-4 u otra IA en un futuro podría ser entrenada con los mismos libros o artículos generados por su predecesor GPT-3; alimentaríamos una IA con datos de otra IA, lo mismo con fotografías o imágenes, pues hoy se generan rostros que nunca han existido mediante Redes Neurales Adversariales (Generative Adversarial Network -GAN). Imagínate leyendo una novela o poesía generada por GPT 3, GPT 4 u otra IA que se haya alimentado con los libros de los mejores escritores. Incluso me invita a pensar si más adelante este artículo podría ser escrito de manera más coherente por una IA. Todo esto supondría reemplazar grandes escritores por algoritmos, aunque ya se hace de manera sencilla en algunos periódicos como el Washington Post o The Guardian (2020) que publicó "A robot wrote this entire article. Are you scared yet, Human?".

GPT-3 y otros modelos de Deep Learning o Machine Learning forman parte de este FST 4.0, el cual podría ser la base de una acelerada y controversial "Modernidad Líquida", concepto creado por el sociólogo Zygmunt Bauman. La razón de ello es que su impacto causará nuevos problemas y cambios sociales, además de reescribir aspectos importantes de nuestra identidad, personalidad, trabajo, educación, actitud individual y colectiva.

¿Encontraríamos una sociedad más caótica 
donde no sepamos si lo que está en internet fue creado por un hombre o una máquina? ¿Qué sentimientos tendrías hacia robots más inteligentes que parecen humanos o tu mascota? ¿En qué medida seremos influenciados o manejados por quienes poseen toda nuestra información? ¿Una melodía, libro o video será generada al 100\% por un algoritmo? Estas preguntas y el surgimiento de este nuevo FST 4.0 nos están conduciendo a un nivel de desarrollo nunca antes visto y en el que vamos a deliberar sobre temas como la renta básica universal, la singularidad tecnológica y el valor humano.

\section{Ejemplo de un texto generado por una IA}

El siguiente texto ha sido generado mediante el uso de la red neuronal Megatron-1B de la empresa InfertKit. Se introdujo la siguiente palabra: "Technology is changing the world" y la IA generó los demás argumentos [1]. Con otra IA se tradujo el texto al español y adicionalmente se generó un rostro humano para la escritora ficticia [2]. Lo siguiente ha sido creación de una IA.

\section{La tecnología está cambiando el mundo}

La velocidad del cambio se ha acelerado desproporcionadamente. Para nosotros, la tecnología no es una moda; es cómo tomamos decisiones en todos los niveles de una empresa y puede determinar si una empresa o un país sobrevive a largo plazo. Un gran ejemplo de una empresa que desafió el embate de la tecnología y logró no solo sobrevivir sino prosperar en un entorno cada vez más desafiante es Apple. Su combinación de productos y sistema operativo de clase mundial con un tremendo valor de marca y capacidades de marketing y ventas inigualables le ha permitido a la empresa diferenciarse de sus pares y seguir creciendo a pesar de la aparición de Internet.

La tecnología también está creando nuevas oportunidades para las organizaciones. Los bancos tradicionales están teniendo que reconsiderar sus estrategias y formas de trabajo de banca comercial y minorista, ya que todos los modelos comerciales se ven interrumpidos por la creciente popularidad de la banca móvil y en línea. Los bancos que sean capaces de poner a sus clientes en primer lugar y la tecnología detrás de ellos prosperarán. Es por eso que vemos este boom tecnológico como una enorme oportunidad para la banca.

Los clientes buscarán un banco que tenga la actitud adecuada, los valores adecuados y la tecno- logía adecuada para brindar un mejor servicio. Pedirán la promesa de sencillez. Querrán hacer cosas que los hagan sentir bien; y esperarán que sus bancos estén disponibles donde quieran realizar operaciones bancarias con ellos. La banca se centra cada vez más en las relaciones y menos en los productos.

Busque bancos y líderes bancarios que operen de una manera cada vez más ágil y receptiva, y cuyos clientes estén satisfechos y bien atendidos. Con mucho ruido en torno a las nuevas tecnologías y servicios digitales, es fácil pasar por alto estos otros atributos. Sigue siendo cierto que los bancos seguirán obteniendo la mayor parte de sus beneficios de sus productos y servicios tradicionales. Sin embargo, la razón principal por la que muchos líderes bancarios no se consideran empresas de tecnología es que están limitados por no ser lo suficientemente ágiles.

Una de las mayores fortalezas de un banco es su gente, y si bien es posible que este comentario cliché se escuche mucho, en el mundo de la banca realmente debe tener pasión por el negocio. Nuestra experiencia con firmas fintech de éxito y sus fundadores es que sienten pasión por el negocio y los clientes. Muchas de estas empresas no tienen mucha experiencia en tecnología, pero sus empresarios tienen una visión y la capacidad de comunicar esta visión a sus clientes.

El propósito de la banca es diferente hoy de lo que era hace diez años. La naturaleza del comportamiento de los clientes está evolucionando. El uso de la tecnología está afectando la forma en que los clientes eligen realizar operaciones bancarias. Los bancos están teniendo que repensar sus productos, estrategias y modelos de servicios. Los bancos tienen que cambiar para sobrevivir en este entorno competitivo.

No es de extrañar que algunas empresas de tecnología financiera estén luchando por comprender por qué los bancos siguen teniendo éxito y no son el mejor lugar para que los emprendedores comiencen y hagan crecer sus negocios. No pueden comprender que personas como mi amigo Kiran, a quien conocí en un evento de oratoria en Mumbai, elijan realizar operaciones bancarias con un banco debido al valor que ofrece. "Simplemente se siente bien", dijo. Como ella me explicó, "Usted gana dinero para mí todos los meses y tiene la tecnología para respaldar su efectivo". En efecto, los bancos todavía tienen la lealtad, la confianza y la relevancia que son raras en el espacio tecnológico. 


\section{Referencias}

Arroyo Gonzales, C. (2006). Modos de Investigar los Fenómenos Sociales. Punto Cero, 11(12), 35-42. http://www.scielo.org.bo/pdf/rpc/v11n12/v11n12a04.pdf

Corvalan, J. G. (2018). Artificial intelligence: Challenges and opportunities-prometea: The first artificial intelligence of Latin America at the service of the justice system. Revista de Investigações Constitucionais, 295. https://doi.org/10.53d80/rinc.v5i1.55334

The Guardian. (8 de Setiembre de 2020). A robot wrote this entire article. Are you scared yet, human? The Guardian. https://www. theguardian.com/commentisfree/2020/sep/08/rob ot-wrote-this-article-gpt-3

Oppenheimer, A. (2018). ¡Sálvese Quién Pueda! El futuro del Trabajo en la Era de la Automatización. Penguin Random House.

\section{Anexo [1] Texto original en inglés}

Technology is changing the world. The speed of change has accelerated out of all proportion. For us, technology is not a fad; it is how we make decisions at every level of a business, and it can determine whether a company or a country survives in the long term. A great example of a company that defied the onslaught of technology and managed to not just survive but thrive in an ever more challenging environment is Apple. Its combination of worldclass products and operating system with tremendous brand equity and unrivalled marketing and sales capabilities has enabled the company to stand apart from its peers and continue to grow despite the emergence of the Internet.

Technology is also creating new opportunities for organisations. Traditional banks are having to reconsider their retail and business banking strategies and ways of working, as all business models are being disrupted by the increasing popularity of mobile and online banking. Banks that are able to put their customer first and technology behind them will thrive. That's why we see this tech boom as an enormous opportunity for banking.

Customers will look for a bank that has the right attitude, the right values, and the right technology to deliver better service. They will ask for the promise of simplicity. They will want to do things that make them feel good; and they will expect their banks to be available wherever they want to bank with them. Banking is becoming more and more about relationships and less about products.
Look for banks and banks leaders that are operating in an ever more agile and responsive way, and whose customers are satisfied and well looked after. With a lot of noise around new technology and digital services, it is easy to overlook these other attributes. It is still the case that banks will still make most of their profits from their traditional products and services. However, the main reason many banking leaders don't consider themselves technology companies is that they are limited by not being nimble enough.

One of the biggest strengths of a bank is its people, and while you might find this clichéd comment heard a lot, in the world of banking you really do need to have a passion for the business. Our experience of successful fintech firms and their founders is that they do have a passion for the business and the customers. Many of these firms do not have much technology expertise, but their entrepreneurs have a vision and an ability to communicate this vision to their customers.

The purpose of banking is different today than it was even 10 years ago. The nature of customers' behaviour is evolving. The use of technology is affecting how customers choose to bank. Banks are having to rethink their products, strategy, and service models. Banks are having to change in order to survive in this competitive environment.

It's no surprise that some fintech firms are struggling to understand why banks remain successful and are not the best place for entrepreneurs to start and grow their businesses. They can't quite fathom that people like my friend Kiran, whom I met at a public speaking event in Mumbai, choose to bank with a bank because of the value that it provides. "It just feels right", she said. As she explained to me, "You are making money for me every month and you have the technology to back up your cash." In effect, banks still have the loyalty, trust, and relevance that is rare in the technology space.

\section{Anexo [2]}

Imagen de la autora ficticia generada con Al.

\section{Cómo citar este artículo:}

Mori Castro, K. (2021). Inteligencia Artificial y sociedad ¿El fenómeno social tecnológico 4.0? Futuro Hoy, 2(1), 3741. https://doi.org/10.5281/zenodo.4654911

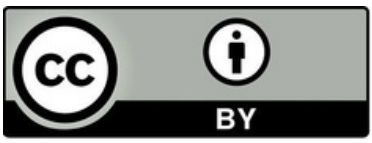

